\title{
Psychosocial interventions for Alzheimer's disease cognitive symptoms: a Bayesian network meta-analysis
}

\author{
Yuting Duan ${ }^{1 \dagger}$, Liming Lư ${ }^{1 \dagger}$, Juexuan Chen ${ }^{1}$, Chunxiao Wu', Jielin Liang ${ }^{1}$, Yan Zheng ${ }^{1}$, Jinjian Wu',
} Peijing Rong ${ }^{2^{*}}$ and Chunzhi Tang ${ }^{1 *}$ (D)

\begin{abstract}
Background: Alzheimer disease (AD) is the most common type of dementia with cognitive decline as one of the core symptoms in older adults. Numerous studies have suggested the value of psychosocial interventions to improve cognition in this population, but which one should be preferred are still matters of controversy. Consequently, we aim to compare and rank different psychosocial interventions in the management of mild to moderate AD with cognitive symptoms.

Methods: We did a network meta-analysis to identify both direct and indirect evidence in relevant studies. We searched MEDLINE, EMBASE, PsycINFO through the OVID database, CENTRAL through the Cochrane Library for clinical randomized controlled trials investigating psychosocial interventions of cognitive symptoms in patients with Alzheimer disease, published up to August 31, 2017. We included trials of home-based exercise(HE), group exercise(GE), walking program(WP) , reminiscence therapy(RT), art therapy(AT) or the combination of psychosocial interventions and acetylcholinesterase inhibitor (ChEls). We extracted the relevant information from these trials with a predefined data extraction sheet and assessed the risk of bias with the Cochrane risk of bias tool. The outcomes investigated were Mini-Mental State Examination (MMSE) and compliance. We did a pair-wise meta-analysis using the fixed-effects model and then did a random-effects network meta-analysis within a Bayesian framework.

Results: We deemed 10 trials eligible, including 682 patients and 11 treatments. The quality of included study was rated as low in most comparison with Cochrane tools. Treatment effects from the network meta-analysis showed WP was better than control (SMD 4.89, 95\% Cl -0.07 to 10.00) while cognitive training and acetylcholinesterase inhibitor (CT + ChEls) was significantly better than the other treatments, when compared with simple ChEls treatment, assessed by MMSE. In terms of compliance, the pair-wise meta-analysis indicated that WP and HE are better than GE and AT, while CT + ChEls, CST + ChEls are better than other combined interventions.
\end{abstract}

Conclusion: Our study confirmed the effectiveness of psychosocial interventions for improving cognition or slowing down the progression of cognitive impairment in AD patients and recommended several interventions for clinical practice.

Keywords: Psychosocial intervention, Alzheimer's disease, Cognitive symptom, Network meta-analysis

\footnotetext{
* Correspondence: drrongpj@163.com; jordan664@163.com

†Yuting Duan and Liming Lu contributed equally to this work.

${ }^{2}$ Institute of Acupuncture and Moxibustion, China Academy of Chinese

Medical Sciences, Beijing, China, 16 Nanxiaojie of Dongzhimen, Beijing

100700, China

${ }^{1}$ Medical College of Acu-Moxi and Rehabilitation, Guangzhou University of

Chinese Medicine, No.232 Waihuandong Road, Panyu District, Guangzhou

510000, China
}

(c) The Author(s). 2018 Open Access This article is distributed under the terms of the Creative Commons Attribution 4.0 International License (http://creativecommons.org/licenses/by/4.0/), which permits unrestricted use, distribution, and reproduction in any medium, provided you give appropriate credit to the original author(s) and the source, provide a link to the Creative Commons license, and indicate if changes were made. The Creative Commons Public Domain Dedication waiver (http://creativecommons.org/publicdomain/zero/1.0/) applies to the data made available in this article, unless otherwise stated. 


\section{Background}

Alzheimer's disease (AD), the most common form of dementia, is characterized by progressive synaptic loss, dysfunction, neuronal death, and vascular toxicity triggered by the deposition of pathologic inducers of lesions in the brain tissue, amyloid $\beta$ peptide, and hyperphosphorylated tau protein [1]. Pharmacological interventions attempting to counteract the lesions have yet to achieve permanent successful results [2-4]. Apart from unsatisfactory efficacy, pharmacological treatments are expensive and have a series of adverse effects.

As an alternative, scientists have turned to non-pharmacological therapies, with psychosocial therapies being one of the most commonly used. In recent years, the publication of several high-quality meta-analyses, systematic reviews, and randomized controlled trials has increased the overall quality of evidence that psychosocial interventions improve or maintain cognition, function, adaptive behavior, and quality of life. For example, Epperly et al. have concluded that cognitive stimulation programs benefit the maintenance of cognitive function and improve self-reported quality of life in patients with mild to moderate Alzheimer's disease [5]. Natasha Yuill et al. have shown that cognitive stimulation therapy is a supportive, functionally-oriented strategy aimed at enabling individuals with mild to moderate dementia to remain meaningfully engaged in their lives and surroundings [6]. The findings of Linda Clare et al. support the clinical efficacy of cognitive rehabilitation in the early stages of AD [7]. However, the 2014 American Psychiatric Association's practice guidelines indicate that the available research has not conclusively determined whether any one intervention is more effective than any other. It also has not conclusively determined which intervention works best for which service setting, specific behavior, disease stage, or caregiver and patient profile [8].

Cognitive impairments are AD's core clinical symptoms, and they impose the greatest burden on patients and their caregivers. Improving patients' cognitive function can delay hospitalization, and therefore reduce the costs of national healthcare and improve both patients' and caregivers' well-being [9]. In order to offer high-quality evidence for clinical decisions, we performed a Bayesian network meta-analysis to compare and rank different psychosocial interventions in the management of cognitive symptoms in patients with AD.

\section{Methods}

This study was conducted in accordance with the Cochrane Handbook for the Systematic Review of Interventions (see details at http://training.cochrane.org/handbook) and the Preferred Reporting Items for Systematic Review and
Meta-Analyses [10]. Included studies were classified according to the type of psychosocial intervention.

\section{Search strategy}

For the two network meta-analyses, we searched MEDLINE, EMBASE and PsycINFO through the OVID database, and searched CENTRAL through the Cochrane Library. We searched studies published from inception to August 31, 2017, and compared any psychosocial interventions for cognitive symptoms in patients with $\mathrm{AD}$ (Additional file 1).

\section{Study selection}

Inclusion criteria and exclusion criteria are summarized in Table 1.

We included the following psychosocial interventions with usual care as the control: home-based exercise (HE), group exercise (GE), walking programs (WP), reminiscence therapy (RT), and art therapy (AT). We also included the combination of psychosocial interventions and ChEIs with ChEIs as a positive control: the combination of cognitive stimulation treatment and acetylcholinesterase inhibitor (CST + ChEIs), the combination of mindfulness-based Alzheimer's stimulation and acetylcholinesterase inhibitor (MBAS+ChEIs), the combination of progressive muscle relaxation and acetylcholinesterase inhibitor (PMR + ChEIs), and the combination of cognitive training and acetylcholinesterase inhibitor (CT + ChEIs).

\section{Data extraction and quality assessment}

Three investigators (YTD, LLM, JXC) independently selected the studies. The review of the main reports and supplementary materials, the extractions of the relevant information from the included trials with a predetermined data extraction sheet, and the assessments of the risk of bias with the Cochrane risk of bias tool were independently performed by three investigators (CXW, JLL, YZ). Any disagreements were resolved through discussion. When they did not reach a consensus, the final decision about each question was made by other investigators within the review team (JJW, CZT, PJR).

We evaluated the quality of the included studies with the Cochrane Collaboration Recommendations assessment tool. The tool for assessing 7 domains, including random sequence generation, allocation concealment, blinding of participants and personnel, blinding (or masking) of outcomes assessors, incomplete outcome data, selective reporting and other bias is described in the Cochrane Handbook for Systematic Reviews of Interventions (see details at http://training.cochrane.org/handbook). Based on these items, studies in which the key domains were all low-risk were considered low-risk, while the remainder were deemed high-risk or unclear-risk, depending on the number of key domains of high or unclear risk. 
Table 1 Eligibility Criteria PICOS

\begin{tabular}{lll}
\hline Inclusion Criteria & Exclusion Criteria \\
\hline Participants & $\begin{array}{l}\text { Meet the diagnosis of National Institute of Neurological and } \\
\text { Communication Disorders and Stroke/Alzheimer's Disease and } \\
\text { Related Disorders Association (NINCDS/ADRDA) criteria or The } \\
\text { Diagnostic and Statistical Manual of Mental Disorders (DSM). }\end{array}$ & $\begin{array}{l}\text { Mild cognitive impairment or other types of non-AD dementia; } \\
\text { familial AD initiated before 50 y old or related to other genetic } \\
\text { diseases. }\end{array}$ \\
$\begin{array}{ll}\text { Interventions } \\
\text { Any type of psychosocial interventions or combination of } \\
\text { psychosocial interventions and ChEls. }\end{array}$ \\
$\begin{array}{ll}\text { Outcomes } \\
\text { Study design }\end{array}$ & Randomized controlled trials; sample size>10/arm. \\
\hline
\end{tabular}

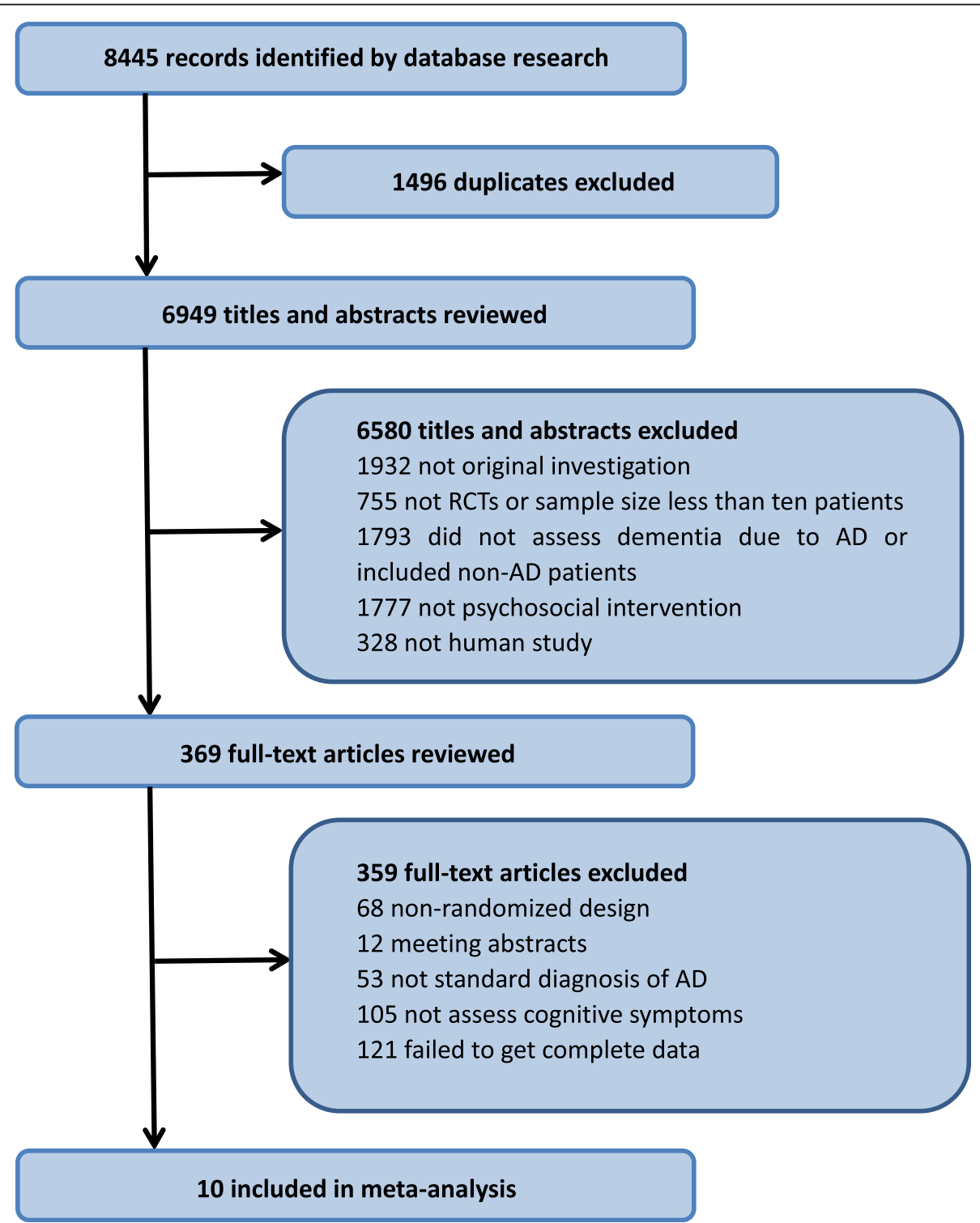

Fig. 1 Study Selection 


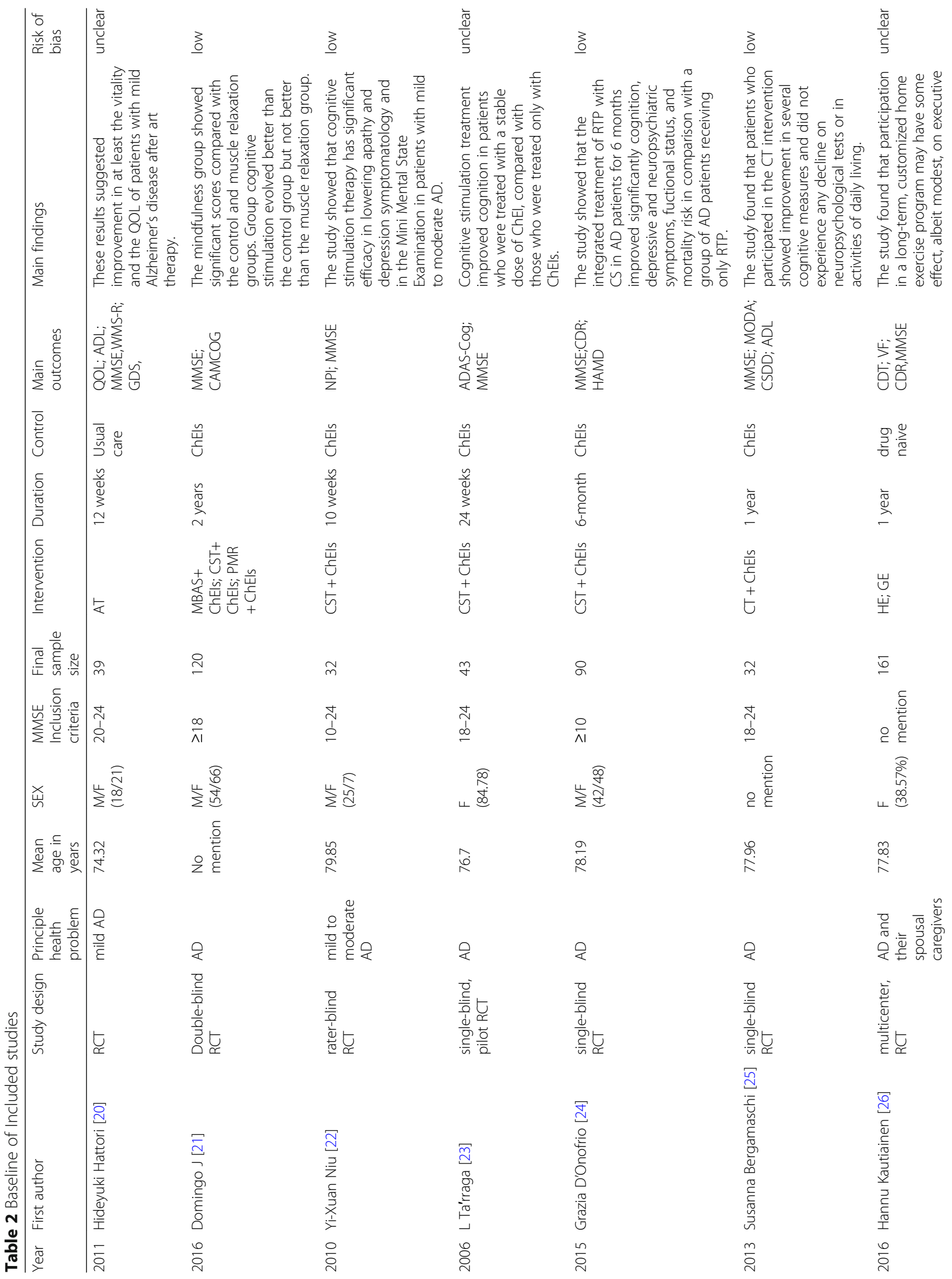


Duan et al. BMC Geriatrics (2018) 18:175

Page 5 of 11

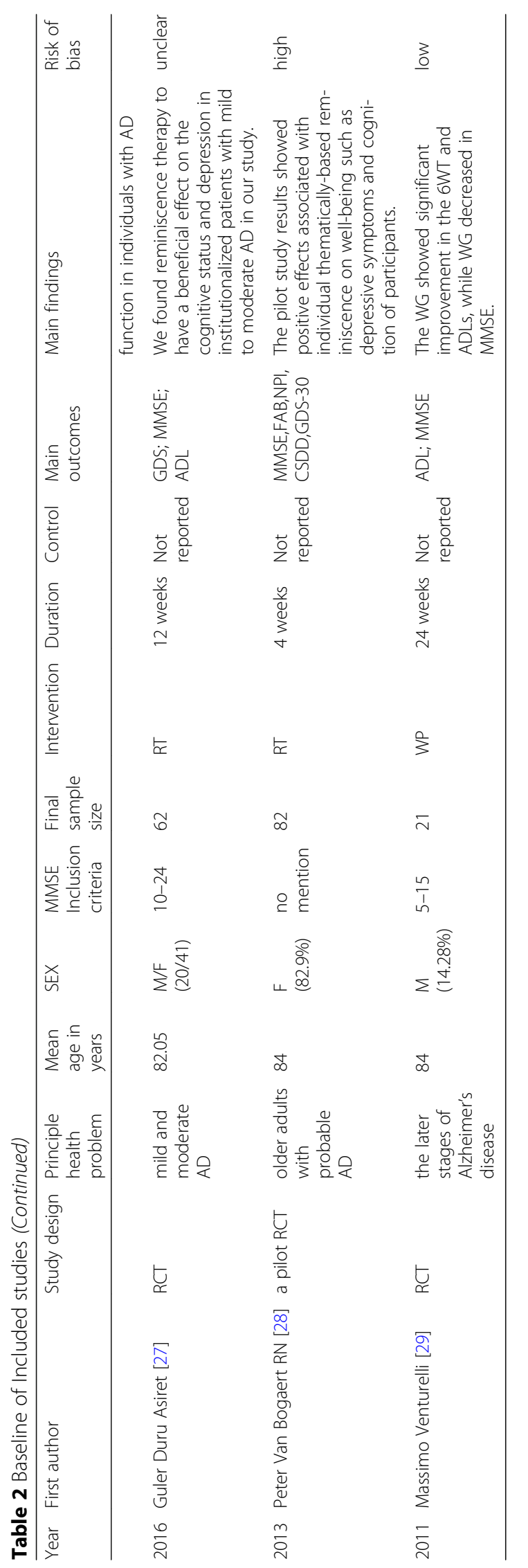




\section{Statistical analysis}

A network meta-analysis with a Bayesian framework with Aggregate Data Drug Information System (ADDIS, version 1.16.8) was conducted to assess the cognitive outcomes of psychosocial interventions. This software is based on the Bayesian framework and the Markov chain Monte Carlo method which can evaluate a priori and process research data. We used a random-effects model to analyze the effect sizes in this study. The effect sizes for continuous outcomes were the mean difference (MD). Consistency and inconsistency were the two models used to estimate the effect size in ADDIS. A consistency assessment drew conclusions on effect sizes of the included interventions and estimated the ranking probabilities for all the interventions. The consistency test was judged by node-splitting analysis and an inconsistence model. When the $p$-value of the node-splitting analysis was greater than 0.05, a consistency mode was selected [11]. Otherwise, an inconsistency model was used. Potential scale reduction factor (PSRF) was used to evaluate the convergence of the model. The closer the PSRF value was to 1 , the better the convergence. The convergence of the model was still acceptable if the PSRF value was less than 1.2. For each intervention, we estimated the ranking probabilities for each treatment at each possible rank.

We ran pair-wise meta-analyses to compare the compliance of different psychosocial therapies because the data included in our study were insufficient for statistical analysis of the network meta-analysis. We conducted the pair-wise meta-analysis with the fixed-effects model with Review Manager (RevMan, v 5.3). The odds ratio (OR) was calculated for dichotomous outcomes (compliance), with 95\% credible intervals (CI). We assessed statistical heterogeneity in

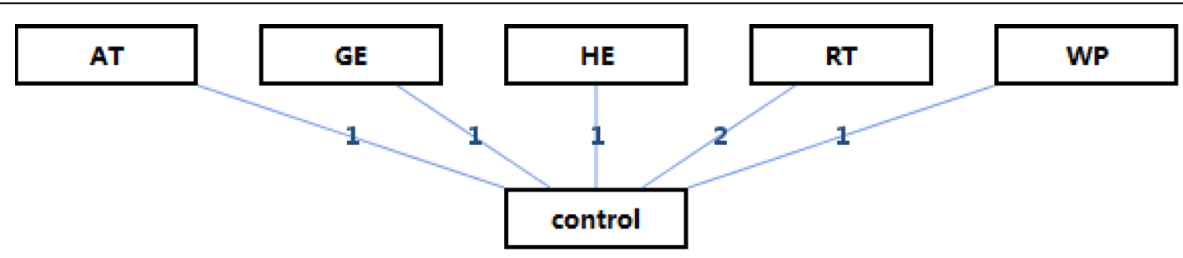

\begin{tabular}{lllllll}
\hline \multicolumn{1}{c}{ Drug } & Rank 1 & Rank 2 & Rank 3 & Rank 4 & Rank & Rank 6 \\
\hline AT & 0.01 & 0.02 & 0.03 & 0.05 & 0.09 & 0.79 \\
GE & 0.03 & 0.07 & 0.21 & 0.33 & 0.27 & 0.09 \\
HE & 0.03 & 0.08 & 0.48 & 0.18 & 0.16 & 0.08 \\
RT & 0.09 & 0.71 & 0.12 & 0.04 & 0.02 & 0.01 \\
WP & 0.83 & 0.10 & 0.03 & 0.02 & 0.01 & 0.01 \\
contro1 & 0.00 & 0.01 & 0.12 & 0.38 & 0.45 & 0.03 \\
\hline
\end{tabular}

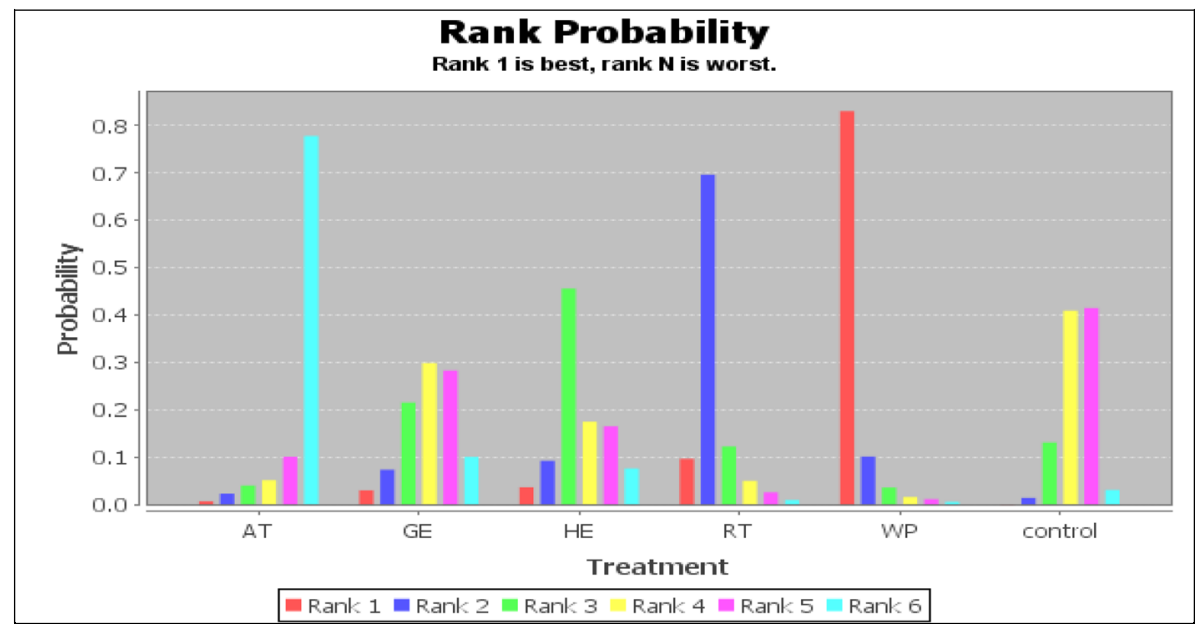

Fig. 2 Rank Probability of Cognitive Effect of the Psychosocial Interventions. AT art therapy, GE group exercise, HE home-based exercise, RT reminiscence, therapy, WP walking program. Control = usual care 
the pair-wise comparison with an $\mathrm{I}^{2}$ statistic and the $p$-value.

\section{Results}

\section{Study identification and selection}

In total, 8445 citations published between 1981 and August 31, 2017 was identified by the search. After removing duplicates and unrelated articles, 10 articles describing 11 RCTs including 682 patients were eligible for further quantitative analyses. A flow chart of the specific screening procedures is shown in Fig. 1. The baseline characteristics of the studies were also extracted (Table 2).

\section{Quality assessment of included studies}

We evaluated the quality of included studies with the Cochrane Collaboration Recommendations assessment tools [12]. Among 10 trials, 8 studies (80\%) described a random component in the sequence generation process such as a computer-generated random number or a random number table. The others did not permit judgment of 'low risk' or 'high risk' due to insufficient information about the sequence generation process. Allocation concealment was performed using an appropriately sealed method in $70 \%$ (7) of the studies, while $30 \%$ (3) either did not describe concrete methods or used an inappropriate allocation concealment method. In performance bias, 70\% (7) of the included trials reported the methods of blinding for both participants and personnel. In detection bias, $30 \%$ (3) of the outcome assessors in the studies either could not be blinded or were unclear. In attrition bias, 9 studies were deemed to have low-risk outcome data (i.e. reported dropout rates within the range of statistical estimations, provided detailed explanations of dropout rates or performed intention-to-treat analysis). 1 study did not provide adequate information to judge the risk of missing data. Other risks were unclear due to insufficient information in 5 studies. Overall, 1 study was considered high risk and 5 were considered low risk, while 4 were considered unclear risk. A detailed quality assessment is presented in Additional files 2 and 3.

\section{Meta-analyses}

A network meta-analysis was performed to compare and rank the included psychosocial interventions which used usual care as their control. The network of eligible comparisons for efficacy consisted of 6 studies and 5 treatments ( 2 arms of RT; 1 arm of AT, GE, HE, WP; 6 arms of control). The specific network is presented in Fig. 2a. The consistency model was selected for the subsequent network analyses. Meanwhile, the inconsistency model was used to test consistency. The results of the network meta-analysis for the primary outcomes are presented as a league table in Table 3. In terms of efficacy, WP was better than the control (SMD 4.98, 95\% CI -0.07 to 10.00 ). This was the best psychosocial intervention for improving cognitive symptoms assessed by the Mini-Mental State Examination (MMSE). The results indicated that WP was significantly more effective than the other treatments in our study. The second and third most effective interventions were RT and HE. The ranking probability of treatments is presented in Fig. $2 \mathrm{~b}$ and c

Consisting of 5 studies and 5 treatments $(2$ arms of CST + ChEIs; 1 arm of CT + ChEIs, MBAS+ChEIs, RMP + ChEIs; 5 arms of ChEIs), another network meta-analysis was run to assess the effectiveness of the combination of psychosocial interventions with ChEIs and simple ChEIs treatment. The specific network is presented in Fig. 3a. In

Table 3 Network Meta-Analysis of Cognitive Effect of Psychosocial Interventions

\begin{tabular}{|c|c|c|c|c|c|}
\hline \multicolumn{6}{|c|}{ Consistency Model of Psychosocial Interventions } \\
\hline AT & $2.48(-4.43,9.24)$ & $2.86(-4.16,9.50)$ & $4.84(-1.29,10.78)$ & $7.29(-0.04,14.07)$ & $2.29(-2.81,7.27)$ \\
\hline$-2.48(-9.24,4.43)$ & GE & $0.39(-6.67,6.84)$ & $2.37(-3.44,8.07)$ & $4.78(-2.48,11.61)$ & $-0.16(-4.99,4.46)$ \\
\hline$-2.86(-9.50,4.16)$ & $-0.39(-6.84,6.67)$ & $\mathrm{HE}$ & $2.00(-3.76,7.77)$ & $4.37(-2.14,11.10)$ & $-0.55(-5.17,4.14)$ \\
\hline$-4.84(-10.78,1.29)$ & $-2.37(-8.07,3.44)$ & $-2.00(-7.77,3.76)$ & RT & $2.38(-3.41,8.34)$ & $-2.53(-5.89,0.69)$ \\
\hline$-7.29(-14.07,0.04)$ & $-4.78(-11.61,2.48)$ & $-4.37(-11.10,2.14)$ & $-2.38(-8.34,3.41)$ & WP & $-4.89(-10.00,0.07)$ \\
\hline$-2.29(-7.27,2.81)$ & $0.16(-4.46,4.99)$ & $0.55(-4.14,5.17)$ & $2.53(-0.69,5.89)$ & $4.89(-0.07,10.00)$ & control \\
\hline \multicolumn{6}{|c|}{ Inconsistency Model of Psychosocial Interventions } \\
\hline AT & $2.44(-4.28,9.85)$ & $2.81(-3.92,9.76)$ & $4.79(-1.16,10.97)$ & $7.24(0.38,14.65)$ & $2.26(-2.56,7.52)$ \\
\hline$-2.44(-9.85,4.28)$ & GE & $0.39(-6.56,6.68)$ & $2.34(-3.71,7.94)$ & $4.74(-2.15,11.43)$ & $-0.16(-4.90,4.38)$ \\
\hline$-2.81(-9.76,3.92)$ & $-0.39(-6.68,6.56)$ & $\mathrm{HE}$ & $1.99(-3.98,7.67)$ & $4.39(-2.58,11.46)$ & $-0.54(-5.39,4.44)$ \\
\hline$-4.79(-10.97,1.16)$ & $-2.34(-7.94,3.71)$ & $-1.99(-7.67,3.98)$ & RT & $2.37(-3.54,8.61)$ & $-2.53(-5.91,1.06)$ \\
\hline$-7.24(-14.65,-0.38)$ & $-4.74(-11.43,2.15)$ & $-4.39(-11.46,2.58)$ & $-2.37(-8.61,3.54)$ & WP & $-4.94(-9.90,0.04)$ \\
\hline$-2.26(-7.52,2.56)$ & $0.16(-4.38,4.90)$ & $0.54(-4.44,5.39)$ & $2.53(-1.06,5.91)$ & $4.94(-0.04,9.90)$ & control \\
\hline
\end{tabular}



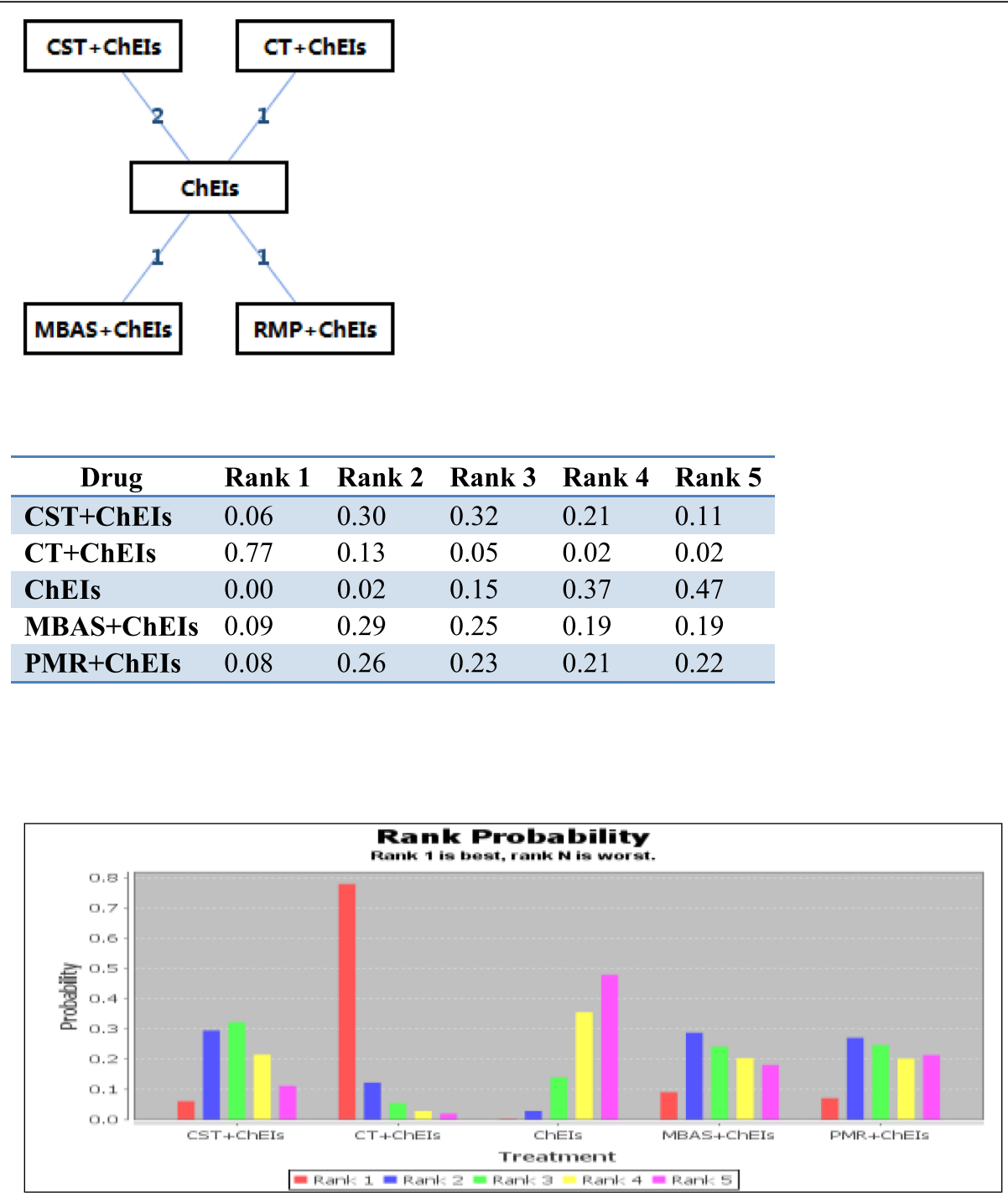

Fig. 3 Rank Probability of Cognitive Effect of the Combination of Psychosocial Interventions and ChEls. CST + ChEls = the combination of cognitive stimulation treatment and acetylcholinesterase inhibitor; $C T+C h E l s=$ the combination of cognitive training and acetylcholinesterase inhibitor; ChEls = acetylcholinesterase inhibitor; MBAS+ChEls = the combination of progressive muscle relaxation and acetylcholinesterase inhibitor; $\mathrm{PMR}+$ ChEls $=$ the combination of progressive muscle relaxation and acetylcholinesterase inhibitor

this subsequent network analysis, we used a consistency model, while an inconsistency model was used to test consistency. The results of the network meta-analyses for the primary outcomes are presented as a league table in Table 4. Note that the curative effect, CT + ChEIs, the best combined intervention for improving cognitive symptoms assessed by MMSE, was better than the control (SMD 6.27, 95\%CI -1.05 to 13.44 ). These results suggested that $\mathrm{CT}+\mathrm{ChEIs}$ was significantly more effective than the other treatments. The second and third most effective interventions were CST + ChEIs and MBAS+ChEIs. The three most effective combined interventions were better than the ChEIs. The ranking probability of treatments is presented in Fig. 3b and c.
Additional file 4 shows the results of pair-wise meta-analyses of compliance for each intervention. The included studies which were not analyzed did not have missing data due to patient non-compliance. We can conclude that patients' compliance with RT, WP and HE are better than GE and AT, and that patients' compliance with CT + ChEIs, CST + ChEIs were better than those of MBAS+ChEIs, RMP + ChEIs and ChEIs.

\section{Discussion}

These findings regarding the comprehensive network meta-analysis represent the most comprehensive synthesis of data for currently available psychosocial AD treatments including the combined treatments of psychosocial 
Table 4 Network Meta-Analysis of Cognitive Effect of the Combination of Psychosocial Interventions and ChEls

\begin{tabular}{lllll}
\hline Consistency Model of Psychosocial Interventions & & & \\
CST + ChEls & $4.51(-4.51,13.02)$ & $-1.73(-7.07,3.33)$ & $-0.09(-9.03,8.71)$ & $-0.29(-9.56,8.33)$ \\
$-4.51(-13.02,4.51)$ & $C T+$ ChEls & $-6.27(-13.44,1.05)$ & $-4.60(-14.92,5.85)$ & $-4.82(-15.10,5.75)$ \\
$1.73(-3.33,7.07)$ & $6.27(-1.05,13.44)$ & ChEls & $1.70(-5.64,9.04)$ & $1.43(-6.00,8.76)$ \\
$0.09(-8.71,9.03)$ & $4.60(-5.85,14.92)$ & $-1.70(-9.04,5.64)$ & MBAS + ChEls & $-0.26(-11.00,10.26)$ \\
$0.29(-8.33,9.56)$ & $4.82(-5.75,15.10)$ & $-1.43(-8.76,6.00)$ & $0.26(-10.26,11.00)$ & PMR + ChEls \\
Inconsistency Model of Psychosocial Interventions & & & $-0.23(-9.24,8.39)$ \\
CST + ChEls & $4.58(-4.49,13.47)$ & $-1.74(-6.90,3.37)$ & $-0.12(-8.89,8.93)$ & $-4.90(-14.98,5.24)$ \\
$-4.58(-13.47,4.49)$ & $C T+$ ChEls & $-6.36(-13.47,0.90)$ & $-4.73(-14.64,5.67)$ & $1.48(-5.74,8.52)$ \\
$1.74(-3.37,6.90)$ & $6.36(-0.90,13.47)$ & ChEls & $1.66(-5.65,8.86)$ & $-0.18(-10.27,9.63)$ \\
$0.12(-8.93,8.89)$ & $4.73(-5.67,14.64)$ & $-1.66(-8.86,5.65)$ & MBAS + ChEls & PMR + ChEls \\
$0.23(-8.39,9.24)$ & $4.90(-5.24,14.98)$ & $-1.48(-8.52,5.74)$ & $0.18(-9.63,10.27)$ & \\
\hline
\end{tabular}

therapies and ChEIs. We found that the cognitive effects of WP, RT, HE and GE are better than the usual care. The combination of the following psychosocial interventions and ChEIs are more effective than ChEIs: CT + ChEIs, CST + ChEIs, MBAS+ChEIs and PMR + ChEIs.

Our study supplements the recommendations of existing guidelines and identifies specific psychosocial interventions with better effects. When only using psychosocial interventions for AD patients, WP and RT showed better efficacy in the management of cognitive symptoms. WP is a physical activity, which is a type of stimulation-oriented intervention. Several clinical investigations have shown its cognitive benefits for older individuals who are healthy, but with mild cognitive impairment or dementia [13-16]. When WP is applied, the adverse reactions of its application should be taken into consideration. For personalized needs, the frequency and intensity of WP should be targeted adjustment. Reminiscence therapy is defined as emotion-oriented interventions in which an individual remembers a past event, verbally or nonverbally, alone or with a group. Reminiscence therapy is another commonly used non-pharmacological application for $\mathrm{AD}$ and other types of dementia which benefits cognition and mental health [17-19].

We also compared and ranked the combination of psychosocial interventions and ChEIs and single used ChEIs, which are the most common used pharmacological interventions. Although there was not enough evidence to prove that psychosocial interventions were better than pharmacological interventions, our study found that the combination of psychosocial interventions and pharmacological interventions was better than treatment with single drugs. Among the included treatments, CT programs combined with pharmacological treatments that could protect patients from functional deterioration by slowing progressive decline showed the best efficacy for cognitive impairments in $\mathrm{AD}$ patients. This revealed that clinical practitioners can try combined therapy, rather than only using drugs.
Evidence indicates that pharmacological treatments for $\mathrm{AD}$ can benefit patients, but important side effects have led to the development of non-pharmacological interventions and their widespread use. We confirmed that the efficacy of psychosocial therapies and combined interventions are better than pharmacological therapies used alone. However, the long-term benefits and the potential for translating these approaches into practice remain uncertain. Meanwhile, the cost-effectiveness also needs to be evaluated with regards to the high education fees for caregivers, as well as the economic burdens for society.

There are several limitations to this review and the data used for the meta-analysis. First, the quality of the included studies was not optimal. When evaluating these studies, we found that many lacked details on randomization or blinding, especially for psychosocial interventions that were difficult to blind. Additionally, several studies had high dropout rates, due inevitably to the length of the trials. Second, although we evaluated the studies according to the tool, any evaluation of bias is subjective. There is no quantitative index that can evaluate only artificial risk of bias. Third, because we used strict inclusion and exclusion criteria, the amount of included studies was less. This may have influenced the strength of the evidence. Fourth, there was no unified index for the classification of intervention methods. Therefore, we categorized them by the descriptions in the literature.

\section{Conclusions}

Our study confirmed the effectiveness of psychosocial interventions for improving cognition or slowing the progression of cognitive impairment in $\mathrm{AD}$ patients. It also recommended several interventions for clinical practice. Future research should be conducted to confirm the impact of psychosocial therapy on other $\mathrm{AD}$ symptoms and additional high-quality RCTs should be performed to provide more powerful evidence. Furthermore, the cost-effectiveness of psychosocial interventions will have to be analyzed. 


\section{Additional files}

Additional file 1: Search Strategy. (DOCX $20 \mathrm{~kb}$ )

Additional file 2: Risk of Bias Graph. (DOCX 31 kb)

Additional file 3: Risk of Bias Summary. (DOCX 25 kb)

Additional file 4: Pair-wise meta-analysis of Compliance for Each Intervention. (DOCX 218 kb)

\section{Abbreviations}

AD: Alzheimer disease; ADDIS: Aggregate Data Drug Information System; AT: Art therapy; ChEls: Acetylcholinesterase inhibitor; Cl: Credible intervals; CST + ChEls: The combination of cognitive stimulation treatment and acetylcholinesterase inhibitor; CT + ChEls: And the combination of cognitive training and acetylcholinesterase inhibitor; GE: Group exercise; HE: Homebased exercise; MBAS+ChEls: the combination of mindfulness-based Alzheimer's stimulation and acetylcholinesterase inhibitor; MMSE: Mini-Mental State Examination; OR: Odds ratio; PMR + ChEls: The combination of progressive muscle relaxation and acetylcholinesterase inhibitor; PSRF: Potential scale reduction factor; RevMan: Review Manager; RT: Reminiscence therapy; WP: Walking program

\section{Acknowledgments}

We thank Benjamin Ross PhD from University of Chicago for language help the authors also express their gratitude for all the researchers who kindly provided the data for the network meta-analysis.

\section{Funding}

Our study is supported by National Natural Science Foundation (Project No. 8167151331) and Guangdong Natural Science Foundation (Project No. 2016A030310290)

\section{Authors' contributions}

YTD, LML, JXC, CZT and PJR conceived and designed the study. YTD, LML, JXC and JLL selected the articles and extracted the data. YZ, JJW, CZT, PJR analyzed the data. YTD, LML and JXC wrote the first draft of the manuscript. CXW, JLL, CZT and PJR interpreted the data and wrote the final version. All authors read and met the ICMJE criteria for authorship and agree with the results and conclusions of the articles.

\section{Ethics approval and consent to participate}

Not applicable.

\section{Consent for publication}

Not applicable.

\section{Competing interests}

The authors declare that they have no competing interests

\section{Publisher's Note}

Springer Nature remains neutral with regard to jurisdictional claims in published maps and institutional affiliations.

\section{Received: 21 February 2018 Accepted: 20 July 2018}

Published online: 07 August 2018

\section{References}

1. Dubois B, Hampel H, Feldman HH, Scheltens P, Aisen P, Andrieu S, Bakardjian H, Benali H, Bertram L, Blennow K, et al. Preclinical Alzheimer's disease: definition, natural history, and diagnostic criteria. Alzheimers Dement. 2016:12(3):292-323.

2. Karran E, Mercken M, De Strooper B. The amyloid cascade hypothesis for Alzheimer's disease: an appraisal for the development of therapeutics. Nat Rev Drug Discov. 2011;10(9):698-712.

3. Salloway S, Sperling R, Fox NC, Blennow K, Klunk W, Raskind M, Sabbagh M, Honig LS, Porsteinsson AP, Ferris S, et al. Two phase 3 trials of bapineuzumab in mild-to-moderate Alzheimer's disease. N Engl J Med. 2014;370(4):322-33.
4. Vandenberghe R, Rinne JO, Boada M, Katayama S, Scheltens P, Vellas B, Tuchman M, Gass A, Fiebach JB, Hill D, et al. Bapineuzumab for mild to moderate Alzheimer's disease in two global, randomized, phase 3 trials. Alzheimers Res Ther. 2016;8(1):18.

5. Epperly T, Dunay MA, Boice JL. Alzheimer disease: pharmacologic and nonpharmacologic therapies for cognitive and functional symptoms. Am Fam Physician. 2017;95(12):771-8.

6. Yuill $\mathrm{N}$, Hollis V. A systematic review of cognitive stimulation therapy for older adults with mild to moderate dementia: an occupational therapy perspective. Occup Ther Int. 2011;18(4):163-86.

7. Clare L, Linden DE, Woods RT, Whitaker R, Evans SJ, Parkinson CH, van Paasschen J, Nelis SM, Hoare Z, Yuen KS, et al. Goal-oriented cognitive rehabilitation for people with early-stage Alzheimer disease: a single-blind randomized controlled trial of clinical efficacy. Am J Geriatr Psychiatry. 2010;18(10):928-39.

8. Guideline watch (October 2014): practice guidline for the treatment of patients with Alzheimer's disease and other dementias. 2014.

9. Graff MJ, Adang EM, Vernooij-Dassen MJ, Dekker J, Jonsson L, Thijssen M, Hoefnagels WH, Rikkert MG. Community occupational therapy for older patients with dementia and their care givers: cost effectiveness study. Bmj. 2008;336(7636):134-8

10. Liberati A, Altman DG, Tetzlaff J, Mulrow C, Gotzsche PC, loannidis JP, Clarke M, Devereaux PJ, Kleijnen J, Moher D. The PRISMA statement for reporting systematic reviews and meta-analyses of studies that evaluate healthcare interventions: explanation and elaboration. Bmj. 2009;339:b2700.

11. Dias S, Welton NJ, Caldwell DM, Ades AE. Checking consistency in mixed treatment comparison meta-analysis. Stat Med. 2010;29(7-8):932-44.

12. Savovic J, Weeks L, Sterne JA, Turner L, Altman DG, Moher D, Higgins JP. Evaluation of the Cochrane Collaboration's tool for assessing the risk of bias in randomized trials: focus groups, online survey, proposed recommendations and their implementation. Systematic reviews. 2014;3:37.

13. Sofi F, Valecchi D, Bacci D, Abbate R, Gensini GF, Casini A, Macchi C. Physical activity and risk of cognitive decline: a meta-analysis of prospective studies. J Intern Med. 2011;269(1):107-17

14. Young J, Angevaren M, Rusted J, Tabet N. Aerobic exercise to improve cognitive function in older people without known cognitive impairment. Cochrane Database Syst Rev. 2015;(4):Cd005381. https://doi.org/10.1002/ 14651858.CD005381.pub4.

15. Law LL, Barnett F, Yau MK, Gray MA. Effects of combined cognitive and exercise interventions on cognition in older adults with and without cognitive impairment: a systematic review. Ageing Res Rev. 2014;15:61-75.

16. Heyn P, Abreu BC, Ottenbacher KJ. The effects of exercise training on elderly persons with cognitive impairment and dementia: a meta-analysis. Arch Phys Med Rehabil. 2004;85(10):1694-704.

17. Irazoki E, Garcia-Casal JA, Sanchez-Meca J, Franco-Martin M. Efficacy of group reminiscence therapy for people with dementia. Systematic literature review and meta-analysis. Rev Neurol. 2017:65(10):447-56.

18. Yen HY, Lin L. A systematic review of reminiscence therapy for older adults in Taiwan. JNR: The journal of nursing research. 2017.

19. Lin HC, Yang YP, Cheng WY, Wang JJ. Distinctive effects between cognitive stimulation and reminiscence therapy on cognitive function and quality of life for different types of behavioural problems in dementia. Scand J Caring Sci. 2017. https://doi.org/10.1111/scs.12484.

20. Hattori H, Hattori C, Hokao C, Mizushima K, Mase T. Controlled study on the cognitive and psychological effect of coloring and drawing in mild Alzheimer's disease patients. Geriatr Gerontol Int. 2011;11(4):431-7.

21. Quintana-Hernandez DJ, Miro-Barrachina MT, Ibanez-Fernandez IJ, Pino AS, Quintana-Montesdeoca MP, Rodriguez-de Vera B, Morales-Casanova D, PerezVieitez Mdel C, Rodriguez-Garcia J, Bravo-Caraduje N. Mindfulness in the maintenance of cognitive capacities in Alzheimer's disease: a randomized clinical trial. Journal of Alzheimer's disease : JAD. 2016;50(1):217-32.

22. Niu YX, Tan JP, Guan JQ, Zhang ZQ, Wang LN. Cognitive stimulation therapy in the treatment of neuropsychiatric symptoms in Alzheimer's disease: a randomized controlled trial. Clin Rehabil. 2010;24(12):1102-11.

23. Tarraga L, Boada M, Modinos G, Espinosa A, Diego S, Morera A, Guitart M, Balcells J, Lopez OL, Becker JT. A randomised pilot study to assess the efficacy of an interactive, multimedia tool of cognitive stimulation in Alzheimer's disease. J Neurol Neurosurg Psychiatry. 2006;77(10):1116-21.

24. D'Onofrio G, Sancarlo D, Addante F, Ciccone F, Cascavilla L, Paris F, Elia AC, Nuzzaci C, Picoco M, Greco A, et al. A pilot randomized controlled trial evaluating an integrated treatment of rivastigmine transdermal patch and 
cognitive stimulation in patients with Alzheimer's disease. Int J Geriatr Psychiatry. 2015;30(9):965-75.

25. Bergamaschi S, Arcara G, Calza A, Villani D, Orgeta V, Mondini S. One-year repeated cycles of cognitive training (CT) for Alzheimer's disease. Aging Clin Exp Res. 2013;25(4):421-6.

26. Ohman H, Savikko N, Strandberg TE, Kautiainen H, Raivio MM, Laakkonen $M L$, Tilvis R, Pitkala KH. Effects of exercise on cognition: the finnish Alzheimer disease exercise trial: a randomized, controlled trial. J Am Geriatr Soc. 2016;64(4):731-8.

27. Duru Asiret $G$, Kapucu S. The effect of reminiscence therapy on cognition, depression, and activities of daily living for patients with Alzheimer disease. J Geriatr Psychiatry Neurol. 2016;29(1):31-7.

28. Van BP, Van GR, Tolson D, Wouters K, Engelborghs S, Van der Mussele S. Effects of SolCos model-based individual reminiscence on older adults with mild to moderate dementia due to Alzheimer disease: a pilot study. Journal of the American Medical Directors Association. 2013;14(7):528.e529-13.

29. Venturelli M, Scarsini R, Schena F. Six-month walking program changes cognitive and ADL performance in patients with Alzheimer. American journal of Alzheimer's disease and other dementias. 2011;26(5):381-8.

Ready to submit your research? Choose BMC and benefit from:

- fast, convenient online submission

- thorough peer review by experienced researchers in your field

- rapid publication on acceptance

- support for research data, including large and complex data types

- gold Open Access which fosters wider collaboration and increased citations

- maximum visibility for your research: over $100 \mathrm{M}$ website views per year

At $\mathrm{BMC}$, research is always in progress.

Learn more biomedcentral.com/submissions 